\title{
Multi-resolution Analysis of Multi-spectral Palmprints using Hybrid Wavelets for Identification
}

\author{
Dr. H.B. Kekre \\ Senior Professor, Department of \\ Computer Science, \\ Mukesh Patel School of Technology \\ Management and Engineering \\ Mumbai, India
}

\author{
Dr. Tanuja Sarode \\ Associate Professor, Department of \\ Computer Science, Thadomal Shahani \\ College of Engineering \\ Mumbai, India
}

\author{
Rekha Vig \\ Asst. Prof. and Research Scholar, Dept. \\ of Electronics and Telecom., \\ Mukesh Patel School of Technology \\ Management and Engineering \\ Mumbai, India
}

\begin{abstract}
Palmprint is a relatively new physiological biometric used in identification systems due to its stable and unique characteristics. The vivid texture information of palmprint present at different resolutions offers abundant prospects in personal recognition. This paper describes a new method to authenticate individuals based on palmprint identification. In order to analyze the texture information at various resolutions, we introduce a new hybrid wavelet, which is generated using two or more component transforms incorporating both their properties. A unique property of this wavelet is its flexibility to vary the number of components at each level of resolution and hence can be made suitable for various applications. Multi-spectral palmprints have been identified using energy compaction of the hybrid wavelet transform coefficients. The scores generated for each set of palmprint images under red, green and blue illuminations are combined using score-level fusion using AND and OR operators. Comparatively low values of equal error rate and high security index have been obtained for all fusion techniques. The experimental results demonstrate the effectiveness and accuracy of the proposed method.
\end{abstract}

Keywords-Hand-based biometrics; Hybrid wavelet; Multiresolution; Energy compaction, fusion;

\section{INTRODUCTION}

With increasing threat to security there is a grave need for identification in our society. Immense focus is in development of accurate and reliable security systems and to facilitate the development of such systems biometrics is considered as a highly effective automatic mechanism for personal identification. As each human being is unique [1], so are his physical characteristics like fingerprint, palmprint, ear, iris etc. and behavioral characteristics like voice, gait etc. These characteristics are considered as biometrics and can be utilized for such automated personal identification system. Palmprints are becoming more popular over fingerprints as biometrics as they offer more stable and reliable features; even the palmveins [2] are being analysed as separate biometrics. The main objective behind the automated systems is to extract the unique features of the biometrics and not treat the entire biometric-trait for identification. Feature extraction can be done in many possible ways. For palmprints the various approaches that can be used are: 1) Line-based approach: Palm lines are prominent and unique features of a palm. The extraction of these lines by using edge-based detection methods [3] and morphological operations [4] are quite common. 2) Subspace (appearance)-based approach: Principle component analysis (PCA), independent component analysis (ICA) and linear discriminant analysis (LDA) are used and their coefficients are considered as the features [5], [6]. 3) Texture-based approach: In this approach various filters like Gabor filter [7]-[9] and transforms like Discrete Cosine Transform and wavelets [10], [11] etc. are used to analyze texture based information of palmprints. Attempts have been made to combine the features [12] produced by few or all the techniques mentioned above.

The texture features are supposed to contain more information and hence they have been successfully analysed using Gabor filter earlier. There exist verification/identification systems based on extracted features using various transforms such as Fourier Transform [13] (where Fourier range and angle features have been extracted to identify the palm-print image) and [14] (where cepstum coefficients are matched), Karhunen-Loeve transform [5], Discrete Cosine Transform, Walsh Transform [18] etc. It has been observed that different transforms analyze the palmprint in different ways. Attempt has been done by Kekre et. al. [17] to combine more than one transforms to generate hybrid transform and use it for palmprint identification. Properties of many transforms can be combined in hybrid transform and hence the results are better than those obtained using single transform. This was further extended to bi-resolution hybrid wavelets [18] which analyze palmprints at only global and local levels of resolution and were successfully used for identification purpose. In this paper we present multiresolution hybrid wavelets, which provide with palmprint image analysis at local, semi-global (at various levels) and global levels of resolution. Since palmprints have principle lines (less texture) which can be viewed at low resolution, minor lines (medium texture) which can be viewed at various resolutions at medium level and ridges and valleys (high texture) which can only be viewed at high resolution, it becomes essential to analyze the palmprint at various resolutions.

The rest of the paper is organized as follows. Section II describes the generation of Hybrid Wavelet using Kronecker product and Hybrid transform. Section III illustrates the algorithm used in the proposed method for palmprint 
identification for multi-spectral images. In section IV the fusion scheme used to combine scores for multi-spectral palmprints is explained and section $\mathrm{V}$ presents the results obtained using the proposed method. The paper is concluded in section VI.

\section{HYBRID WAVELET}

\section{A. Generation of Hybrid Wavelet}

The hybrid wavelet transform matrix is generated using hybrid transform matrix, which can be produced using Kronecker product of two or more existing orthogonal transforms[15]. For any hybrid transform of size $n \times n=p q x p q$ (component transforms $\mathrm{A}$ and $\mathrm{B}$ of size pxp and qxq respectively), a hybrid wavelet $\mathrm{H}_{\mathrm{W}}$, of size pqxpq can be generated. If $r_{0}, r_{1}, r_{2}, \ldots r_{n-1}$ (except 1 and $p$ itself) are the divisors of $\mathrm{p}$ (for example, if $\mathrm{p}=32$ then $2,4,8$ and 16 will be $r_{0}, r_{1}, r_{2}$ and $r_{3}$ respectively), arranged in ascending order, then the hybrid wavelet is given by,

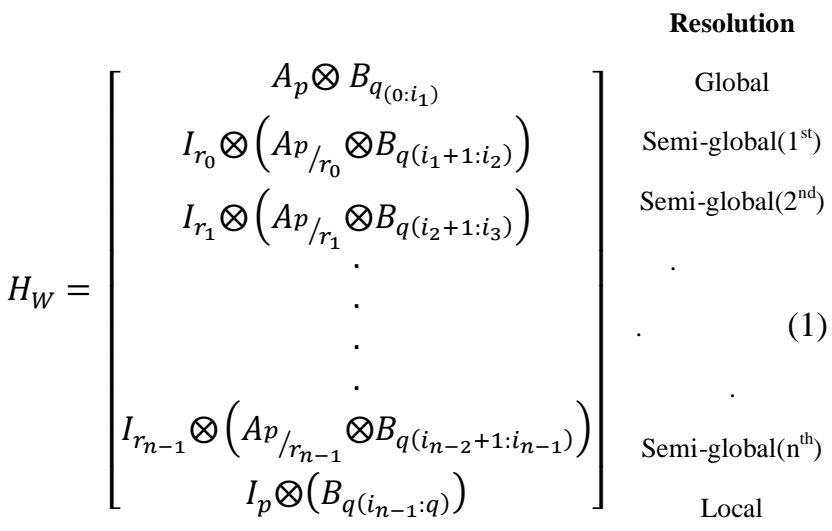

where $B_{q(i: j)}$ represents the rows $i$ to $j$ of $B$ matrix of size $q$ and $A_{p / r}$ represents $A$ matrix of size $p / r$. Here, the transform matrix $A$ of size $p$ and its lower order matrices of sizes $p / r_{0}$, $\mathrm{p} / \mathrm{r}_{1}, \ldots \mathrm{p} / \mathrm{r}_{\mathrm{n}-1}$ are multiplied (using Kronecker product) with certain rows of $\mathrm{B}$ transform matrix. Note that the rows of $\mathrm{B}$ are not repeated. The scaling operation used in the generation of existing wavelets like Haar, has been replaced by making use of lower order matrices of transform matrix A. The shifting operation is being realized by making use of the identity matrix (I) which is multiplied (using Kronecker product) with the lower order hybrid transform components. The lower the order of the transform matrix A, the higher is the order of I matrix by the same factor.

For example, employing the component transforms Walsh and Kekre transform matrices of sizes $p=4$ and $q=3$ (Walsh $4 \mathrm{X} 4$ and Kekre $3 \mathrm{X} 3$ ), a hybrid wavelet can be generated as in equation (2).

$$
H_{W}=\left[\begin{array}{c}
W_{4} \otimes K_{3(0)} \\
I_{2} \otimes\left(W_{4 / 2} \otimes K_{3(1)}\right) \\
I_{4} \otimes\left(K_{3(2)}\right)
\end{array}\right]
$$

which gives

$$
\boldsymbol{H}_{W}=\left[\begin{array}{rrrrrrrrrrrr}
1 & \mathbf{1} & \mathbf{1} & \mathbf{1} & \mathbf{1} & \mathbf{1} & \mathbf{1} & \mathbf{1} & \mathbf{1} & \mathbf{1} & \mathbf{1} & \mathbf{1} \\
\mathbf{1} & \mathbf{1} & \mathbf{1} & \mathbf{1} & \mathbf{1} & \mathbf{1} & -\mathbf{1} & -\mathbf{1} & -\mathbf{1} & -\mathbf{1} & -\mathbf{1} & -\mathbf{1} \\
\mathbf{1} & \mathbf{1} & \mathbf{1} & -\mathbf{1} & -\mathbf{1} & -\mathbf{1} & -\mathbf{1} & -\mathbf{1} & -\mathbf{1} & \mathbf{1} & \mathbf{1} & \mathbf{1} \\
\mathbf{1} & \mathbf{1} & \mathbf{1} & -\mathbf{1} & -\mathbf{1} & -\mathbf{1} & \mathbf{1} & \mathbf{1} & \mathbf{1} & -\mathbf{1} & -\mathbf{1} & -\mathbf{1} \\
-\mathbf{2} & \mathbf{1} & \mathbf{1} & -\mathbf{2} & \mathbf{1} & \mathbf{1} & \mathbf{0} & \mathbf{0} & \mathbf{0} & \mathbf{0} & \mathbf{0} & \mathbf{0} \\
-\mathbf{2} & \mathbf{1} & \mathbf{1} & \mathbf{2} & -\mathbf{1} & -\mathbf{1} & \mathbf{0} & \mathbf{0} & \mathbf{0} & \mathbf{0} & \mathbf{0} & \mathbf{0} \\
\mathbf{0} & \mathbf{0} & \mathbf{0} & \mathbf{0} & \mathbf{0} & \mathbf{0} & -\mathbf{2} & \mathbf{1} & \mathbf{1} & -\mathbf{2} & \mathbf{1} & \mathbf{1} \\
\mathbf{0} & \mathbf{0} & \mathbf{0} & \mathbf{0} & \mathbf{0} & \mathbf{0} & -\mathbf{2} & \mathbf{1} & \mathbf{1} & \mathbf{2} & -\mathbf{1} & -\mathbf{1} \\
\mathbf{0} & -\mathbf{1} & \mathbf{1} & \mathbf{0} & \mathbf{0} & \mathbf{0} & \mathbf{0} & \mathbf{0} & \mathbf{0} & \mathbf{0} & \mathbf{0} & \mathbf{0} \\
\mathbf{0} & \mathbf{0} & \mathbf{0} & \mathbf{0} & -\mathbf{1} & \mathbf{1} & \mathbf{0} & \mathbf{0} & \mathbf{0} & \mathbf{0} & \mathbf{0} & \mathbf{0} \\
\mathbf{0} & \mathbf{0} & \mathbf{0} & \mathbf{0} & \mathbf{0} & \mathbf{0} & \mathbf{0} & -\mathbf{1} & \mathbf{1} & \mathbf{0} & \mathbf{0} & \mathbf{0} \\
\mathbf{0} & \mathbf{0} & \mathbf{0} & \mathbf{0} & \mathbf{0} & \mathbf{0} & \mathbf{0} & \mathbf{0} & \mathbf{0} & \mathbf{0} & -\mathbf{1} & \mathbf{1}
\end{array}\right]
$$

Here the first $\mathrm{p}(=4)$ rows represent global components, the next $\mathrm{p}$ rows represent semi-global and the last $\mathrm{p}$ rows represent local components of the hybrid wavelets.

With A and B as component transforms, hybrid wavelet $\mathrm{H}_{\mathrm{W}}$ can be formed in the following different ways:

1) $\quad p$ and $q$ can take different values for the same value of $n$, both being the factors of $n$.

2) $A$ and $B$ can be interchanged, if the transform matrices of the corresponding sizes exist.

3) The values of $i_{0}$ through $i_{n-1}$ can be varied such that size of $H_{W}$ remains $p q x p q$.

\section{B. Properties of Hybrid Wavelet}

1) Orthogonal: The hybrid wavelet follows the principle of orthogonality i.e.

$$
\mu_{w}=\left[\begin{array}{c}
H_{W} * H_{W}{ }^{T}=\mu_{W} \\
\mu_{A p} \otimes \mu_{B q_{\left(0: i_{1}\right)}} \\
\mu_{A p / r_{1}} \otimes \mu_{B_{q\left(i_{1}+1: i_{2}\right)}} \\
\mu_{A p / r_{2}} \otimes \mu_{B_{q\left(i_{2}+1: i_{3}\right)}} \\
\cdot \\
\cdot \\
\cdot \\
\mu_{A p / r_{n-1}} \mu_{B_{q\left(i_{n-2}+1: i_{n-1}\right)}}
\end{array}\right]
$$

where $\mu_{\mathrm{W}}$ is a diagonal but may not be an identity matrix and $\mu_{\mathrm{A}}$ and $\mu_{\mathrm{B}}$ are the diagonal matrices given by $\mu_{\mathrm{A}}=\mathrm{AA}^{\mathrm{T}}$ and $\mu_{\mathrm{B}}=\mathrm{BB}^{\mathrm{T}}$.

2) Hybrid wavelet coefficients: To calculate the hybrid wavelet coefficients $\mathrm{F}$, of a one-dimensional discrete function $\mathrm{f}$, we use,

$$
F=H_{W} * f
$$

and to obtain the discrete function from the coefficients $F$ we use

$$
f=H_{W}{ }^{T} * \mu_{W}{ }^{-1} * F
$$


The coefficients $\mathrm{D}$ of a two-dimensional function $\mathrm{d}$ are obtained by

$$
D=H_{W} * d * H_{W}{ }^{T}
$$

And the inverse is obtained by first calculating $G$ matrix whose $(\mathrm{i}, \mathrm{j})^{\text {th }}$ element is given by,

$$
G_{i j}=\frac{H_{W_{i j}}}{\mu_{w_{i j}}}
$$

where $\mu_{\mathrm{Wij}_{\mathrm{ij}}}=\mu_{\mathrm{Wii}} * \mu_{\mathrm{W}_{\mathrm{jj}}}$ for all values of $\mathrm{i}$ and $\mathrm{j}$.

Then,

$$
d=G^{T} * D * G
$$

Energy in each transform coefficient $\mathrm{D}$ in frequency domain is given by

$$
E_{i j}=\frac{D_{i j}^{2}}{\mu_{w_{i j}}}
$$

Thus the total energy in the function $\mathrm{d}$ is given by

$$
E=\sum_{i, j=1}^{n} \frac{D_{i j}^{2}}{\mu_{w_{i j}}}=\sum_{i, j=1}^{n} d_{i j}^{2}=\text { input energy }
$$

\section{ALGORITHM FOR PALMPRINT IDENTIFICATION}

The hybrid wavelet developed in the previous section has been used with various component transforms for the purpose of multi-spectral palmprint identification. As transforms and wavelets are known to show energy compaction in the lower frequency coefficients and higher frequency coefficients are minusculed, it is possible to discard these low energy (high frequency) coefficients. This is the basis for the reduction in feature vector size. The algorithm used in the process is as shown in the figure 1.

\section{A. Enrollment phase:}

Each ROI extracted palmprint image is transformed using the hybrid wavelet transform as generated in section II using equation (1). The hybrid wavelet transform is generated using transforms matrices A and B, which can be any of the existing orthogonal transforms like DCT, Walsh, Kekre, etc. The size of the hybrid wavelet matrix is the same as that of the palmprint image used; here the image is $128 \mathrm{X} 128(\mathrm{n}=128)$, the $\mathrm{H}_{\mathrm{W}}$ is of the same size and is generated with values of $\mathrm{p}=$ 16 , and $q=8$ (or any other factor combination like $p=32$ and $q=4$ ). Various component transforms were tried and the best results were obtained using a hybrid wavelet with Walsh and DCT as component transforms.

Energy in each transform coefficient is its magnitude squared and scaled by the energy matrix coefficient, $\mu_{\mathrm{w}_{\mathrm{ij}}}$ of hybrid wavelet as in equation (10). The coefficient energy matrices of all database images are averaged to generate mean energy matrix. This energy matrix is divided into blocks of $4 \times 4$ and mean of each block is calculated, thus reducing the size to $32 \times 32$. This is primarily done to reduce the feature vector size. These mean energy values of mean energy matrix are arranged in descending order of individual component energy and those blocks are selected whose cumulative energy is more than threshold energy. The selected blocks form the Energy Map. The standard deviation values of the transform coefficients of these blocks are the Feature Vector. Higher the threshold energy larger the feature vector size and better the identification rate. But beyond certain threshold energy value, there is negligible increase in the identification rate, though the feature vector size increases considerably.

\section{B. Identification Phase:}

The ROI extracted test palmprint is transformed and its energy matrix is generated. This is also divided into $4 \times 4$ blocks and the mean energy of each block is calculated. Those blocks are selected which map to the energy map as generated in enrollment phase. Then the standard deviation values of the transform coefficients of these blocks of the test palmprint are its features.

\section{Matching criteria:}

Absolute Distance (AD) has been used as a distance measure to compare the FV of test image $\left(\mathrm{FV}_{\mathrm{t}}\right)$ with that of each image in the database $\left(\mathrm{FV}_{\mathrm{d}}\right)$. The $\mathrm{m}_{\mathrm{th}} \mathrm{AD}$ between the test image and $\mathrm{m}_{\mathrm{th}}$ database image is given by equation (12)

$$
A D_{m}=\sum_{i=1}^{s}\left|F V_{t i}-F V_{(d m)_{i}}\right|
$$

where $\mathrm{s}$ is the number of enrolled images. A genuine match is defined as a match between the palmprints from the same palm and an imposter match is the match between the palmprints from different palms. If the $\mathrm{AD}$ is lower than a given threshold value, a match is obtained. This value of threshold is varied to obtain the genuine acceptance ratio (GAR) and false acceptance ratio (FAR). The performance of the proposed method is obtained by plotting the receiver operating characteristics (ROC) and calculating the equal error rate (EER). 


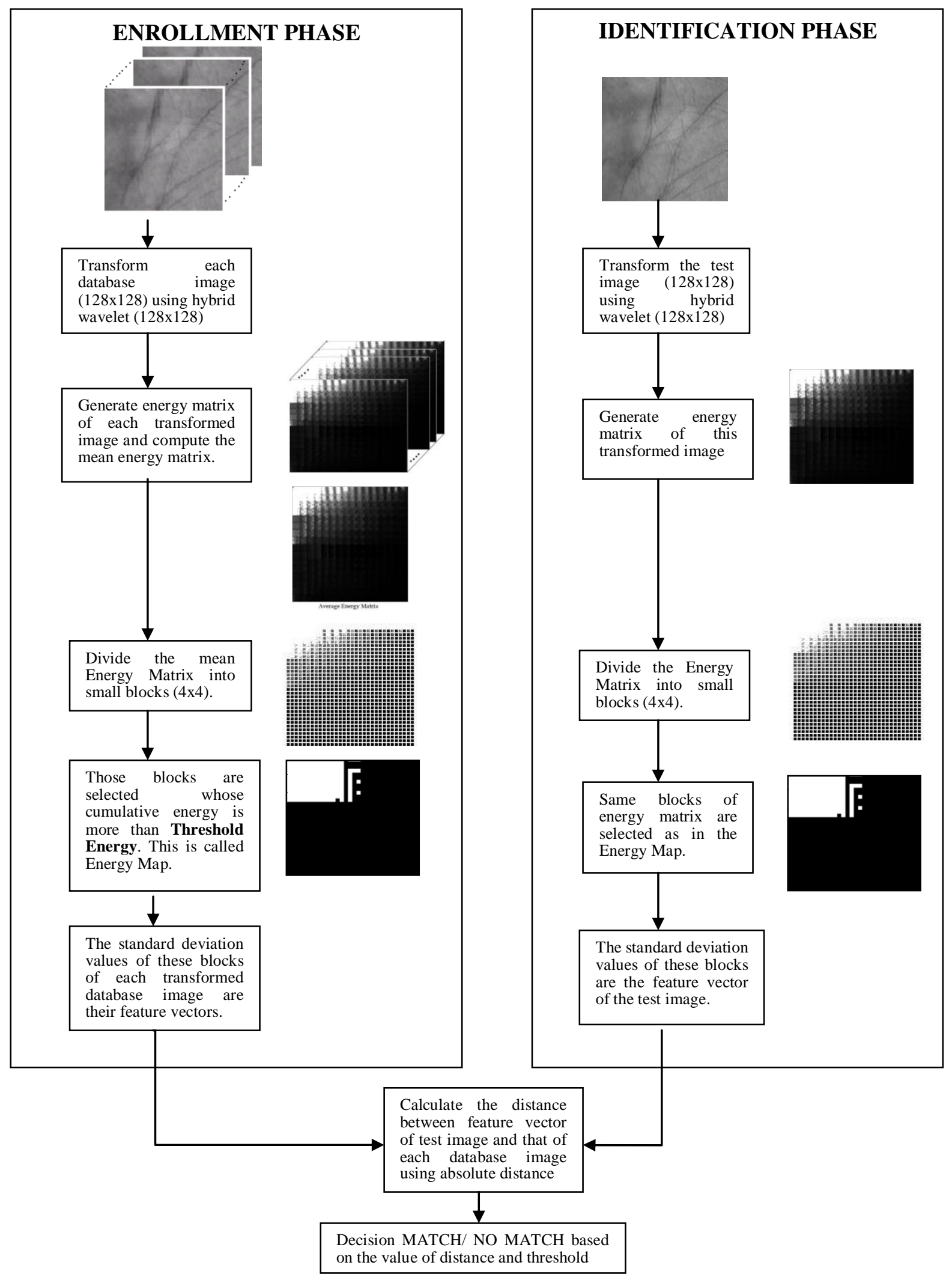

Fig 1. Flow diagram of algorithm for palmprint identification using the proposed method 


\section{Score Level Fusion Scheme}

Fusion of the scores obtained for multi-spectral palmprints under red, green and blue (RGB) illumination is done to combine the characteristics of palmprints under each illumination. The fusion rules aim at improving the performance of a system from that of a single palmprint. Fusion can be done at three general levels: feature level, score level and decision level. The score level fusion of feature sets has been used most commonly and gives significant improvement in performance. In this paper we have used score level fusion for multi-spectral palmprint images to combine the scores produced individually for multispectral palmprint images under red, green and blue illuminations [10] (henceforth referred to as R, G and B images) as shown in Figure 2. Three types of fusions schemes using AND and OR operators are used to combine scores and final matching decision can be taken for either of the three fusion strategies. Considering $S_{R}, S_{G}$ and $S_{B}$ as the scores produced for $R, G$ and $B$ images respectively, they have been combined in the following three ways to obtain the final score $S_{\mathrm{F}}$ :

$$
\begin{aligned}
& \text { I. } \mathrm{S}_{\mathrm{F}}=\mathrm{S}_{\mathrm{R}}{\text { OR } \mathrm{S}_{\mathrm{G}} \text { OR } \mathrm{S}_{\mathrm{B}}}_{\text {II. }} \\
& \mathrm{S}_{\mathrm{F}}=\left\{\mathrm{S}_{\mathrm{R}} \text { AND } \mathrm{S}_{\mathrm{G}}\right\} \text { OR }\left\{\mathrm{S}_{\mathrm{R}} \text { AND } \mathrm{S}_{\mathrm{B}}\right\} \text { OR }\left\{\mathrm{S}_{\mathrm{B}}\right. \text { AND } \\
& \text { III. }\left.\mathrm{S}_{\mathrm{G}}\right\} \\
& \mathrm{S}_{\mathrm{F}}=\mathrm{S}_{\mathrm{R}} \text { AND } \mathrm{S}_{\mathrm{G}} \text { AND } \mathrm{S}_{\mathrm{B}}
\end{aligned}
$$

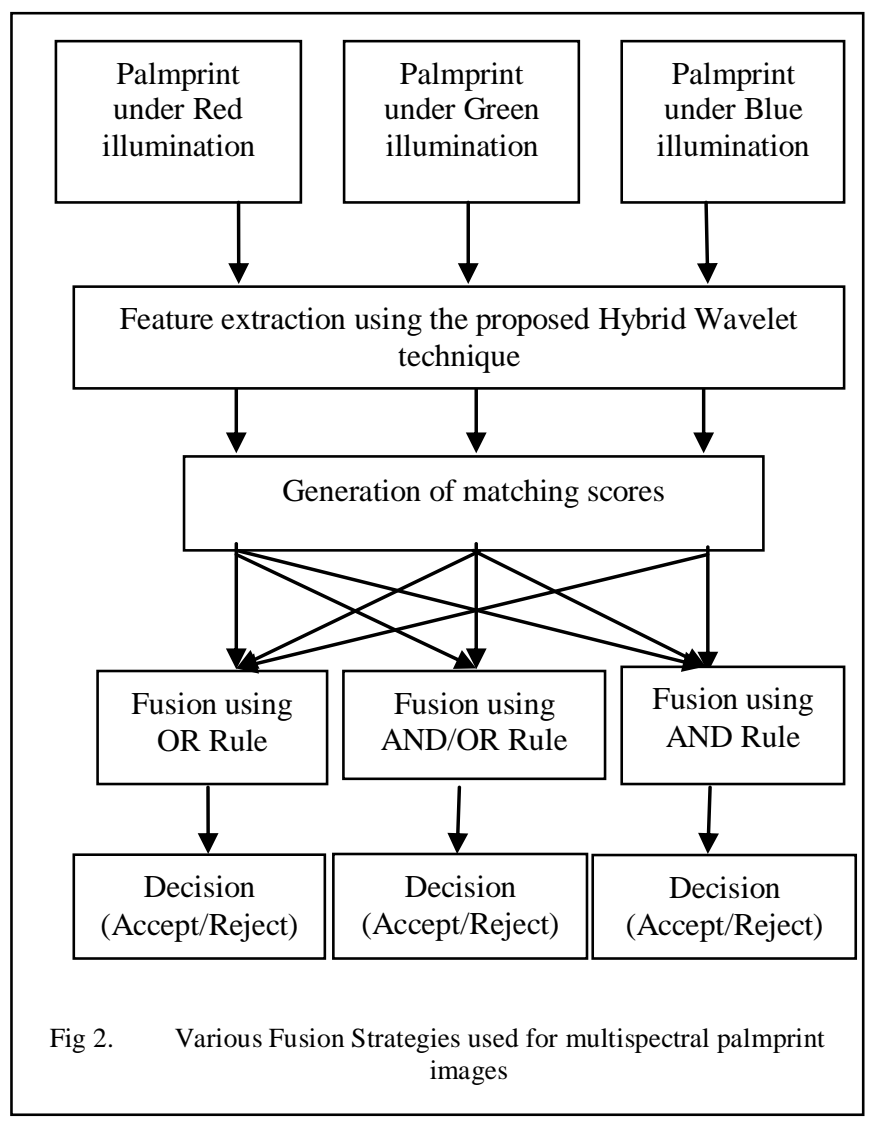

For different applications requiring different levels of security, various score level fusion strategies can be used. The fusion strategy using AND operator is more stringent, as it gives a match only when all R, G, and B images match and hence is used for applications requiring higher level of security whereas OR operator is more liberal in terms of security but better in accuracy. In between these two extremes is the AND/OR based fusion rule which is a compromise between level of security and accuracy.

\section{RESUlTS AND DisCUSSION}

\section{A. Database}

This experiment was performed on the multi-spectral palmprint database of Hongkong Polytechnic University, which consists of multi-spectral palmprint images under R, G and $\mathrm{B}$ illuminations. As different features of palmprints are captured at different wavelengths of light, multi-spectral palmprints are more effective in the analysis. Multispectral imaging in any area is supposed to preserve detailed radiance information and has great discriminative power[19].

The database [16] used here comprises of three sets of 6000 (12 samples of 500 persons) ROI extracted palmprint images of size $128 \times 128$, each under red, green and blue illuminations. For the experiment purpose, each of these sets has been divided into 2 sets: 4800 (12 samples of 400 persons) as enrolled and 1200 (12 samples of 100 persons) as imposters' palmprints. For genuine palmprint identification each of the 4800 palmprints of the enrolled set is individually treated as test image and matched against the remaining 4799 palmprints. For checking imposter identification, each of the imposters' palmprint set is treated as test image and matched against the enrolled set.

\section{B. Results}

For multi-resolution analysis of multi-spectral palmprints, many component transform pairs from DCT, Walsh, Kekre, Haar for generating hybrid wavelet have been tried. The best results obtained are using DCT $(8 \times 8)$ and Walsh $(16 \times 16$ and lower order) as component transform matrices and have been discussed here. The performance of the proposed method has been quantitatively evaluated using False Reject Rate (FRR), False Acceptance Rate (FAR) and Equal Error Rate (EER). For evaluation of FRR, each of the 4800 images of each of the $\mathrm{R}, \mathrm{G}$ and $\mathrm{B}$ set is considered as a test image and identified against the remaining 4799 images. For calculation of FAR, 1200 images of the test set are identified against 4800 images of the training set. Once the matching results are obtained, we get the complete palmprint matching score using rule based score-level fusion. The experimental results show that significant improvement in the performance can be achieved from the combination of scores of multi-spectral palmprint images. Figure 3 shows the plot of GAR vs. FAR for all the three fusion schemes and it can be observed that OR-based fusion scheme gives the best performance for GAR. The ROC plot for the three fusion scheme shown in figure 3, indicate that the EER obtained for OR-based fusion scheme is the least at $1.833 \%$, whereas for AND-based fusion scheme it is $2.604 \%$. The value of threshold (static) at which EER is seen to be lower for OR-based fusion and is 375 and for ANDbased fusion technique the same is 810 . The higher the value of threshold, more secure the system is. Another parameter called security index has been introduced, which can be used to judge the performance of a system with respect to security and can be defined as 


$$
S_{p}=\frac{T_{Z}}{T_{E}}
$$

where, $\mathrm{T}_{\mathrm{Z}}$ is the maximum value of threshold for which FRR is zero and $T_{E}$ is the value of threshold where EER is obtained. $S_{\mathrm{P}}$ is security index and lies between 0 and 1 and if the value is 1 then it is an ideal system in terms of security at which EER becomes zero. For the proposed system, the values of $S_{P}$ for AND and AND-OR based fusion systems are 0.875 and 0.887 respectively and for OR based fusion system it is obtained as 0.853 , clearly indicating that AND based systems are highly secure.

Hence it can be concluded that the proposed method, when used with AND-based fusion technique can be used for applications where high level of security is demanded and ORbased fusion where higher efficiency is required with slight compromise in security.

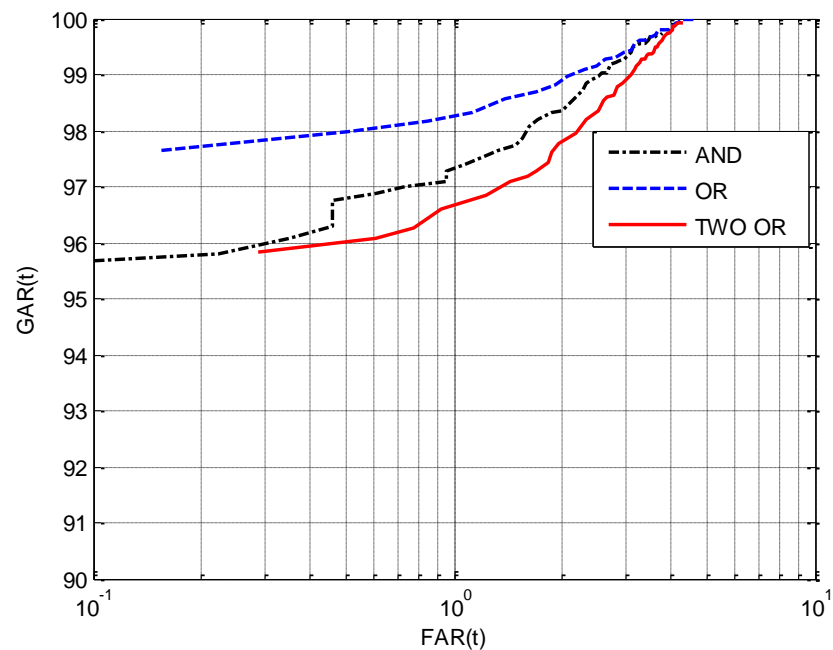

(a)

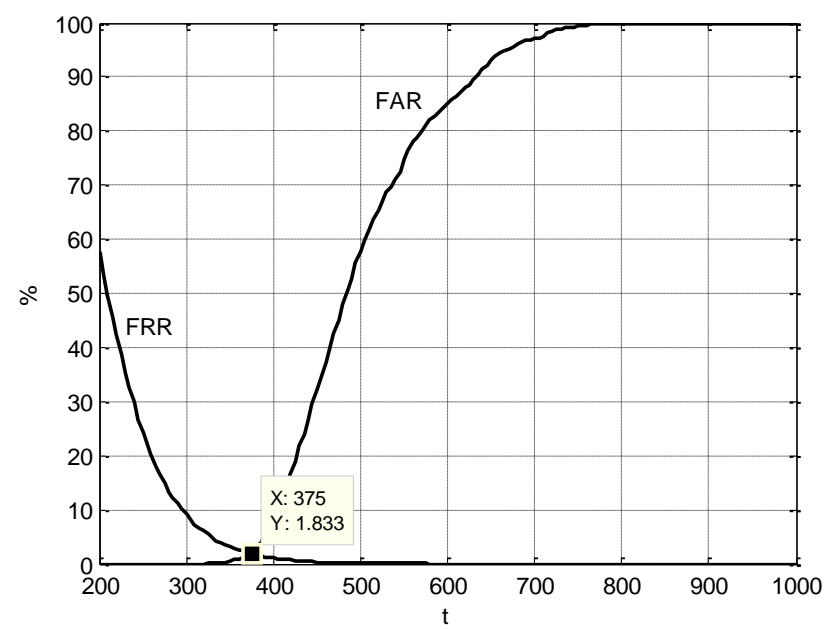

(b)

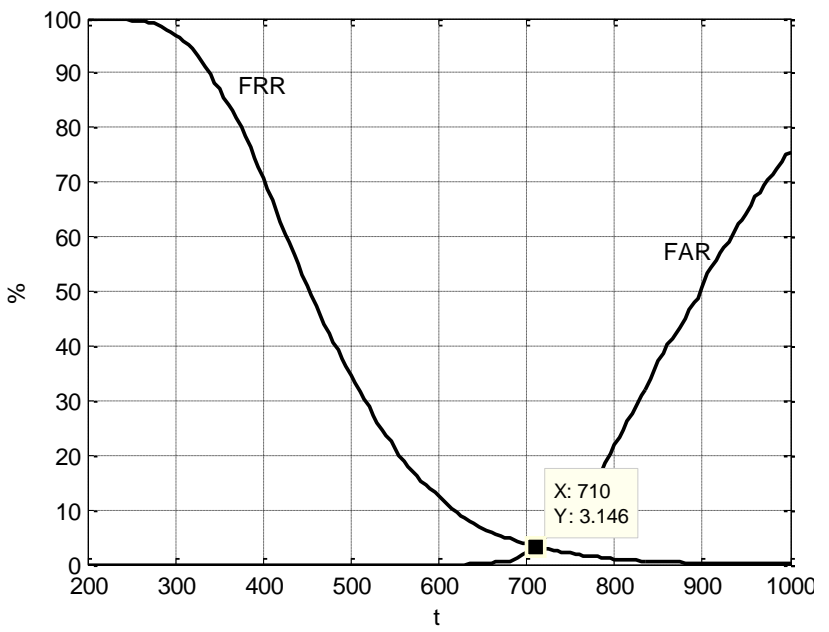

(c)

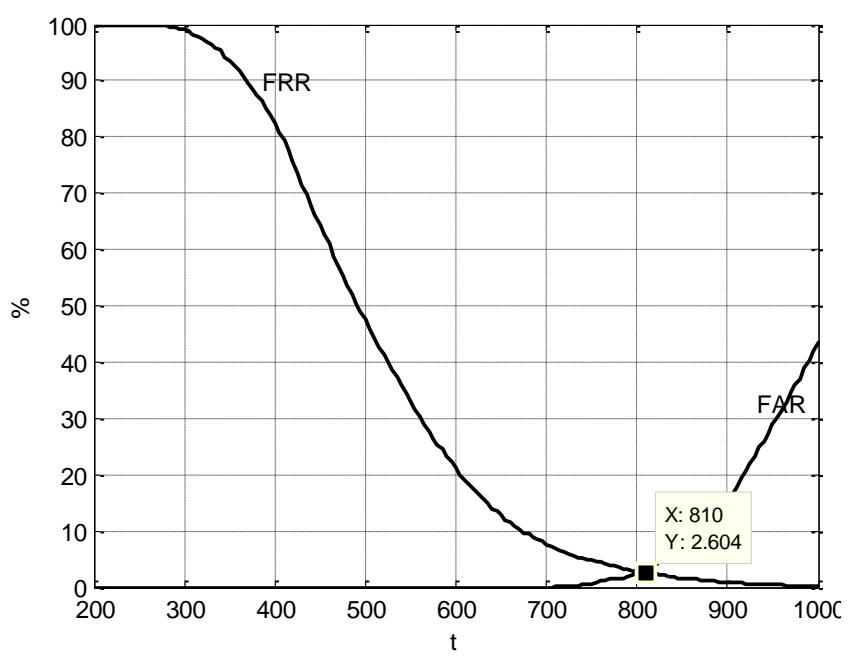

(d)

c) AND-OR based d) AND based fusion

\section{CONCLUSION}

In this paper palmprint identification using a new hybrid wavelet employing multi-resolution analysis is proposed. The hybrid wavelet used here is a flexible wavelet which analyzes functions at all levels of resolutions where number of components at each level can be controlled and the wavelet itself can be varied by using different component transforms. The hybrid wavelet used here gives far better performance than the individual component transforms in various applications like image data compression, content-based image retrieval etc. Multi-spectral palmprints are identified using the features generated by energy compaction of the hybrid wavelet coefficients and fusion of the scores of each set of the red, green and blue images using OR and AND based techniques is used to find best system. The performance of the proposed method is analysed by calculating equal error rate and security performance index and the values of EER of $1.833 \%$ suggests that system using OR-based fusion scheme 
gives better performance efficiency, whereas $S_{P}$ of 0.887 and 0.875 for AND-OR and AND fusion based system respectively indicate that these are highly secured systems. Future scope where hybrid wavelet with more than two different transforms can be used for palmprint identification. Application of this technique for other biometrics is open for further analysis and research.

\section{REFERENCES}

[1] Anil K. Jain, Encyclopedia of Biometrics, Springer Publication, August 27, 2009

[2] Yingbo Zhou and Ajay Kumar. Human Identification Using Palm-Vein Images. IEEE Transactions on Information Forensics and Security, vol. 6(4) 2011, p.1259-1274.

[3] X. Wu, K. Wang and D. Zhang, "Line feature extraction and matching in palmprint", in Proceeding of the Second International Conference on Image and Graphics, 2002, p. 583-590.

[4] C.C. Han, H.L. Cheng, C.L. Lin and K.C. Fan, "Personal authentication using palm-print features", Pattern Recognition, vol. 36, no. 2, 2003, p. 371-381.

[5] Lu, G., David, Z., Wang, K., "Palmprint recognition using eigenpalms features", Pattern Recognition Letters, 24(9-10), 2003, p 1473-1477.

[6] X.Q. Wu, D. Zhang, K. Q. Wang, "Fisherpalms Based Palmprint Recognition", Pattern Recognition Letters, vol. 24, 2003, pp. 2829-2838,

[7] David Zhang, Zhenhua Guo, Guangming Lu, Lei Zhang, and Wangmeng Zuo. An Online System of Multi-spectral Palmprint Verification. IEEE Transactions on Instrumentation and Measurement, vol. 59(2), 2010, p. 480-490,

[8] Dong Han, Zhenhua Guo, and David Zhang. Multispectral Palmprint Recognition using wavelet-based Image Fusion. International Conference on Signal Processing, 2008, p.2074-2077.

[9] David Zhang, Wai-kin Kong, Jane You and Michael Wong. On-line palmprint identification. IEEE Transactions on Pattern Analysis and Machine Intelligence, vol. 25(9) 2003, p.1041-1050.

[10] L. Zhang, Z. Guo, Z. Wang, and D. Zhang. Palmprint Verification using Complex Wavelet Transform. ICIP (2), 2007, p. 417-420,

[11] G. Lu, K. Wang and D. Zhang "Wavelet based feature extraction for palmprint identification", Second International Conference on Image and Graphics, 2002, p. 780-784.

[12] A. Kumar and D. Zhang, "Personal authentication using multiple palmprint representation”, Pattern Recognition, vol. 38, no. 10, 2005, p. 1695-1704,

[13] W. Li, D. Zhang, and Z. Xu. Palmprint Identification by Fourier Transform. Int'l J. Pattern Recognition and Artificial Intelligence, vol. 16(4), 2002, p. 417-432.

[14] Dr. H.B.Kekre, Dr. Tanuja Sarode, Rekha Vig, "Automated Fingerprint Identification System based on Sectorized Complex Walsh Plane", IJCA Proceedings on International Conference on Technology Systems and Management (ICTSM), 2011, p. 6-11.

[15] Dr. H.B.Kekre, Dr. Tanuja Sarode, Rekha Vig. Unified Fast Algorithm for Most Commonly used Transforms using Mixed Radix and Kronecker product. International Journal of Computer Science and Information Security, 2011, p. 271-350.

[16] http://www.comp.polyu.edu.hk/ biometrics/MultispectralPalmprint/MS P.htm

[17] Dr. H.B.Kekre, Dr. Tanuja Sarode, Rekha Vig, Pranay Arya, Aashita Irani, Saurabh Bisani, Palmprint Identification using Kronecker Product of DCT and Walsh Transforms for Multi-spectral Images, International Conference on Hand-Based Biometrics, 2011.

[18] Dr. H.B.Kekre, Dr. Tanuja Sarode, Rekha Vig, Pranay Arya, Aashita Irani, Saurabh Bisani, Identification of Multi-spectral Palmprints using Energy Compaction by Hybrid Wavelet, International Conference on Biometrics, 2011

[19] Zhenhua Guo, David Zhang, Lei Zhang, and Wenhuang Liu. Feature Band Selection for Online Multispectral Palmprint Recognition. IEEE Transactions on Information Forensics and Security, vol. 7(3) 2012, p.1094-1099. 